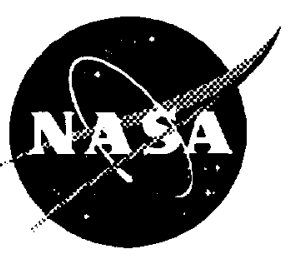

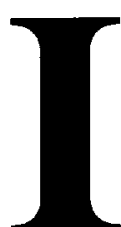
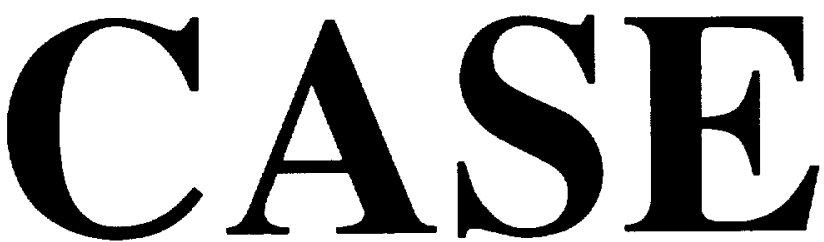

\title{
A SPECTRAL BOUNDARY INTEGRAL EQUATION METHOD FOR THE 2-D HELMHOLTZ EQUATION
}

Fang Q. Hu

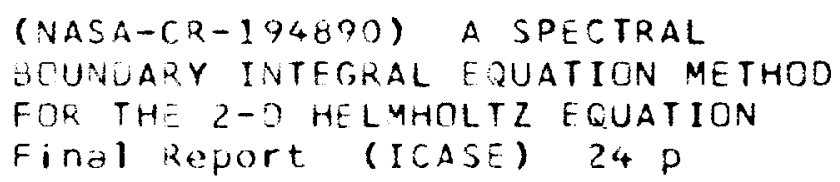

Unclas

Institute for Computer Applications in Science and Engineering NASA Langley Research Center Hampton, VA 23681-0001

SRA Operated by Universities Space Research Association 


\title{
A SPECTRAL BOUNDARY INTEGRAL EQUATION METHOD FOR THE 2-D HELMHOLTZ EQUATION
}

\author{
FANG Q. HU \\ Department of Mathematics and Statistics, Old Dominion University \\ Norfolk, VA 23529
}

\begin{abstract}
In this paper, we present a new numerical formulation of solving the Boundary Integral Equations reformulated from the Helmholtz equation. The boundaries of the problems are assumed to be smooth closed contours. The solution on the boundary is treated as a periodic function, which is in turn approximated by a truncated Fourier series. A Fourier collocation method is followed in which the boundary integral equation is transformed into a system of algebraic equations. It is shown that in order to achieve spectral accuracy for the numerical formulation, the non-smoothness of the integral kernels, associated with the Helmholtz equation, must be carefully removed. The emphasis of the paper is on investigating the essential elements of removing the nonsmoothness of the integral kernels in the spectral implementation. The present method is robust for a general boundary contour. Aspects of efficient implementation of the method using FFT are also discussed. A numerical example of wave scattering is given in which the exponential accuracy of the present numerical method is demonstrated.
\end{abstract}

\footnotetext{
${ }^{1}$ This research was supported by the National Aeronautics and Space Administration under NASA Contract No. NAS1-19480 while the author was in residence at the Institute for Computer Applications in Science and Engineering (ICASE), NASA Langley Research Center, Hampton, VA 23681.
} 



\section{INTRODUCTION}

The Boundary Integral Equation Method is a powerful tool for solving certain boundary value problems. It is particularly attractive in developing numerical methods since, when applied, it reduces the dimension of the problem and often transforms a problem in an infinite domain to integrals on the finite boundary in which the far field radiation condition is satisfied automatically.

Numerical methods for the Boundary Integral Equations have been developed predominantly based on the Boundary Element Method (BEM). In this method the physical boundary is divided into finite elements and integrations over each element are approximated by numerical quadratures. In this way, the integral equation is converted into a system of algebraic equations. BEM has gone through a rapid advancement in recent years. Its applications to the Helmholtz equation are discussed in reference [2] and the references cited therein. Other formulations of solving the Boundary Integral Equations in wave scattering and propagation have also been proposed in the past, including, for example, the T-matrix Method[3,4,5].

In this paper, we present a spectral method formulation for solving the boundary integral equations reformulated from the Helmholtz equation. For the Fourier approximations used in this paper, we assume that the boundary of the problem is a smooth closed contour. The functions appearing in the 2-D boundary integral equation will be treated as periodic functions, which are in turn approximated with high accuracy using truncated Fourier series. The boundary integral equation is then transformed into a system of linear algebraic equations. The spectral methods have been known to have extremely fast convergence rates, faster than any finite power of $1 / N$ when the solution is infinitely smooth[6,7] (where $N$ is the number of collocation points). The present numerical formulation will be seen to have spectral accuracy.

It is known that, although any periodic function can be approximated by a truncated Fourier series, the rate of convergence depends on its smoothness. Unfortunately, the integral kernels for the Helmholtz equation are not smooth. In particular, we note 
that the 2-D Green's function, appearing in the integral equations, possesses a logarithmic singularity. Furthermore, the normal derivative of the Green's function also contains a term involving the logarithmic function. It will be seen that it is critical to remove the non-smoothness of the integral kernel in order to achieve fast convergence in the spectral formulation. In this paper the non-smoothness of the integral kernels is subtracted out by using a logarithmic periodic function whose Fourier expansion is known.

Fourier approximation methods for the boundary integral equations in wave scattering have been proposed in the past for simple geometries. A "fast numerical method" has been formulated by Bojarski[8] for wave scattering by a circular cylinder. It was pointed out that the boundary integral equation for scattering by a hard circular cylinder can be solved easily and efficiently in the Fourier spectrum domain of the solution. Due to the special geometry considered, a simple relation for the Fourier coefficients of the solution and those of the boundary condition was derived. Recently a similar numerical approach has been applied by Schuster[9] for a wave transmission problem of concentric cylinders. However, in these works the singularities and non-smoothness of the integral kernels were not removed. Consequently the convergence rates of these methods were not exponential.

In section 2, a formulation of the Boundary Integral Equation for the Helmholtz equation is given, followed by a discretization using a Fourier collocation method. Essential elements of achieving spectral accuracy are investigated. In section 3, a numerical example is given in which the spectral rate of convergence is demonstrated. Section 4 contains the conclusions.

\section{FORMULATIONS}

\subsection{Boundary Integral Equations}

Consider wave propagation in an interior or exterior domain with a smooth closed boundary $\Gamma$, Figure 1 . The wave equation for a scalar function $\phi$ with assumed time 
dependency of $e^{-i \omega t}$ is reduced to the Helmholtz equation

$$
\nabla^{2} \phi+\kappa^{2} \phi=0
$$

where $\kappa$ is the wave number and $\nabla^{2}$ is the 2-D Laplace operator, $\nabla^{2}=\frac{\partial^{2}}{\partial x^{2}}+\frac{\partial^{2}}{\partial y^{2}}$. The boundary condition considered in this paper is one of the following types :

$$
\begin{aligned}
\text { Dirichlet : } & \phi(\vec{r})=a(\vec{r}) \quad \text { On } \Gamma \\
\text { Neumann : } & \frac{\partial \phi}{\partial n}(\vec{r})=b(\vec{r}) \quad \text { On } \Gamma \\
\text { Robin(Impedance }): & \alpha \phi(\vec{r})+\beta \frac{\partial \phi}{\partial n}(\vec{r})=c(\vec{r}) \quad \text { On } \Gamma
\end{aligned}
$$

The Helmholtz equation (1) can be reformulated into a Boundary Integral Equation in various ways[10,11]. Our purpose in this paper is to demonstrate the numerical method using spectral approximations and the essential elements of achieving spectral accuracy of the numerical solution. A direct formulation of the Boundary Integral Equation employing the Green's Function will be used here which leads to an integral equation involving $\phi$ and its normal derivative on the boundary[2]:

$$
\frac{1}{2} \phi\left(\vec{r}_{\Gamma}^{\prime}\right)+\int_{\Gamma} \phi \frac{\partial G}{\partial n} d \Gamma=\int_{\Gamma} G \frac{\partial \phi}{\partial n} d \Gamma
$$

where $\frac{\partial}{\partial n}$ denotes the normal derivative, with the direction of $\vec{n}$ being outward from the domain of wave propagation, and $\vec{r}_{\Gamma}^{\prime}$ denotes any point on the boundary. The Green's function $G\left(\vec{r}, \vec{r}^{\prime}\right)$, whose form will be given later, satisfies the following equation

$$
\nabla^{2} G+\kappa^{2} G=-\delta\left(\vec{r}-\vec{r}^{\prime}\right)
$$

Once the values of $\phi$ and $\frac{\partial \phi}{\partial n}$ on the boundary are found, the solution at any point, $\vec{r}$, inside the domain of wave propagation can be obtained as [2]

$$
\phi(\vec{r})=\int_{\Gamma}\left(G \frac{\partial \phi}{\partial n}-\phi \frac{\partial G}{\partial n}\right) d \Gamma
$$


Without loss of generality, let us now assume the boundary curve $\Gamma$ be parameterized as

$$
\vec{r}_{\Gamma}=\vec{r}(\theta), \quad 0 \leq \theta \leq 2 \pi
$$

where $\theta$ is a non-dimensional parameter for the boundary contour, not necessary the arclength or some angle. In addition, the curve is supposed to be simple, that is

$$
\left|\frac{d \vec{r}}{d \theta}\right|=\sqrt{\left(\frac{d x}{d \theta}\right)^{2}+\left(\frac{d y}{d \theta}\right)^{2}} \neq 0
$$

For simplicity, we further assume that $\vec{r}(\theta)$ is infinitely differentiable. Then the boundary integral equation (2) can be written as

$$
\frac{1}{2} \phi\left(\theta^{\prime}\right)+\int_{0}^{2 \pi} \phi(\theta) \frac{\partial G}{\partial n}\left(\theta, \theta^{\prime}\right)\left|\frac{d \vec{r}}{d \theta}\right| d \theta=\int_{0}^{2 \pi} \frac{\partial \phi}{\partial n}(\theta) G\left(\theta, \theta^{\prime}\right)\left|\frac{d \vec{r}}{d \theta}\right| d \theta
$$

in which the line element $d \Gamma=\left|\frac{d \vec{r}}{d \theta}\right| d \theta$. For clarity, the dependency on $\theta$ has been expressed explicitly in (5).

For the 2-D Helmholtz equation, the out-going Green's function and its normal derivative are[12] :

$$
\begin{gathered}
G\left(\theta, \theta^{\prime}\right)=\frac{i}{4} H_{0}^{(1)}(\kappa R) \\
\frac{\partial G}{\partial n}\left(\theta, \theta^{\prime}\right)=-\frac{i \kappa}{4} H_{1}^{(1)}(\kappa R) \frac{\vec{R} \cdot \vec{n}}{R}
\end{gathered}
$$

in which we have used the notations that $\vec{R}=\vec{r}(\theta)-\vec{r}\left(\theta^{\prime}\right)$ and $R=|\vec{R}|$. Here $H_{0}^{(1)}$ and $H_{1}^{(1)}$ are the first kind Hankel functions of order zero and one, respectively.

\subsection{Spectral Approximations}

For a closed boundary $\Gamma, \phi(\theta)$ and $\frac{\partial \phi}{\partial n}(\theta)$ are periodic functions of $\theta$, for $0 \leq \theta \leq$ $2 \pi$. Let $\phi$ and $\frac{\partial \phi}{\partial n}$ be approximated by truncated Fourier series as :

$$
\phi^{N}(\theta)=\sum_{n=-N / 2}^{N / 2-1} \hat{\phi}_{n} e^{i n \theta}
$$




$$
\frac{\partial \phi^{N}}{\partial n}(\theta)=\sum_{n=-N / 2}^{N / 2-1} \hat{\psi}_{n} e^{i n \theta}
$$

The particular form of the truncated Fourier series has been taken in (8) and (9) for the convenience of using discrete Fast Fourier Transform (FFT) programs[7]. Substituting (8) and (9) into (5), the boundary integral equation becomes the following

$$
\frac{1}{2} \sum_{n=-N / 2}^{N / 2-1} \hat{\phi}_{n} e^{i n \theta^{\prime}}+\sum_{n=-N / 2}^{N / 2-1} \hat{\phi}_{n} \int_{0}^{2 \pi} e^{i n \theta} \frac{\partial G}{\partial n}\left(\theta, \theta^{\prime}\right)\left|\frac{d \vec{r}}{d \theta}\right| d \theta=\sum_{n=-N / 2}^{N / 2-1} \hat{\psi}_{n} \int_{0}^{2 \pi} e^{i n \theta} G\left(\theta, \theta^{\prime}\right)\left|\frac{d \vec{r}}{d \theta}\right| d \theta
$$

We note that the two integrals in (10) are actually the Fourier coefficients of $G\left(\theta, \theta^{\prime}\right)\left|\frac{d \vec{r}}{d \theta}\right|$ and $\frac{\partial G}{\partial n}\left(\theta, \theta^{\prime}\right)\left|\frac{d \vec{r}}{d \theta}\right|$ respectively. Our aim is to evaluate the integrals by the Fast Fourier Transforms with spectral accuracy.

It is clear that both $G\left(\theta, \theta^{\prime}\right)\left|\frac{d \vec{r}}{d \theta}\right|$ and $\frac{\partial G}{\partial n}\left(\theta, \theta^{\prime}\right)\left|\frac{d \vec{r}}{d \theta}\right|$ are periodic functions of $\theta$ and $\theta^{\prime}$. They are also infinitely differentiable except at $\theta=\theta^{\prime}$ where $R=0$ in (6) and (7). Although any periodic function can be approximated in a truncated Fourier series, the rate of convergence depends on the smoothness of the function. From this consideration, we note that, first, $G\left(\theta, \theta^{\prime}\right)$ has a logarithmic singularity at $R=0$, due to the Hankel function of order zero in (6). Second, although $\frac{\partial G}{\partial n}\left(\theta, \theta^{\prime}\right)$ can be shown to be a finite function, it is not infinitely smooth. It is easy to show that the function $R$ has a discontinuous derivative at $\theta=\theta^{\prime}$. In particular, for $\left|\theta-\theta^{\prime}\right|$ small, we have

$$
\begin{aligned}
R=\left|\vec{r}(\theta)-\vec{r}\left(\theta^{\prime}\right)\right| & =\left|\frac{d \vec{r}}{d \theta} \cdot\left(\theta-\theta^{\prime}\right)+\frac{1}{2} \frac{d^{2} \vec{r}}{d \theta^{2}} \cdot\left(\theta-\theta^{\prime}\right)^{2}+\cdots \cdots\right| \\
& =\left|\theta-\theta^{\prime}\right|\left|\frac{d \vec{r}}{d \theta}+\frac{1}{2} \frac{d^{2} \vec{r}}{d \theta^{2}}\left(\theta-\theta^{\prime}\right)+\cdots \cdots\right|
\end{aligned}
$$

Consequently any term with an odd power or logarithmic function of $R$ will not be infinitely smooth and has to be treated in order to achieve spectral accuracy in the Fourier approximation. 
To study the singularity in $G\left(\theta, \theta^{\prime}\right)$, we note that

$$
G\left(\theta, \theta^{\prime}\right)=\frac{i}{4} H_{0}^{(1)}(\kappa R)=\frac{i}{4}\left(J_{0}(\kappa R)+i Y_{0}(\kappa R)\right)
$$

in which $J_{0}$ and $Y_{0}$ are the zeroth order Bessel functions of the first and second kind, respectively. Using the asymptotic series for a small argument[13],

$$
\begin{aligned}
& J_{0}(\kappa R)=1-\frac{(\kappa R)^{2}}{4}+\frac{(\kappa R)^{4}}{64}-\cdots \\
& Y_{0}(\kappa R)=\frac{2}{\pi} \ln \left(\frac{\kappa R}{2}\right) J_{0}(\kappa R)+\frac{2 \gamma}{\pi} J_{0}(\kappa R)+\frac{(\kappa R)^{2}}{2 \pi}-\cdots
\end{aligned}
$$

we have, for $\left|\theta-\theta^{\prime}\right|$ small,

$$
\begin{aligned}
G\left(\theta, \theta^{\prime}\right) & =\frac{i}{4}\left(J_{0}(\kappa R)+i Y_{0}(\kappa R)\right) \\
& =-\frac{1}{2 \pi} \ln \left(\frac{\kappa R}{2}\right) J_{0}(\kappa R)+(\text { smooth terms })
\end{aligned}
$$

in which "smooth terms" represents a convergent power series containing only the even powers of $R$, and $J_{0}$ is the regular Bessel function of the zeroth order[13], also a power series of $R^{2}$. To remove the logarithmic singularity but preserve the periodicity, consider the periodic function $\ln \left|2 \sin \left(\frac{\theta-\theta^{\prime}}{2}\right)\right|$, which has a logarithmic singularity at $\theta=\theta^{\prime}$. Its Fourier series can be found exactly[12] :

$$
\ln \left|2 \sin \left(\frac{\theta-\theta^{\prime}}{2}\right)\right|=-\sum_{n=1}^{\infty} \frac{\cos n\left(\theta-\theta^{\prime}\right)}{n}
$$

To subtract out the singularity in $G\left(\theta, \theta^{\prime}\right)$, let

$$
\bar{G}\left(\theta, \theta^{\prime}\right) \equiv \frac{i}{4} H_{0}^{(1)}(\kappa R)+\frac{1}{2 \pi} \ln \left|2 \sin \left(\frac{\theta-\theta^{\prime}}{2}\right)\right| J_{0}(\kappa R)
$$

Then the Green's function is found as

$$
G\left(\theta, \theta^{\prime}\right)=\bar{G}\left(\theta, \theta^{\prime}\right)-\frac{1}{2 \pi} \ln \left|2 \sin \left(\frac{\theta-\theta^{\prime}}{2}\right)\right| J_{0}(\kappa R)
$$

Using (11), it is easy to show that $\bar{G}\left(\theta, \theta^{\prime}\right)$ is periodic but finite for all values of the arguments. Furthermore both $\bar{G}\left(\theta, \theta^{\prime}\right)$ and $J_{0}(\kappa R)$ are now infinitely differentiable 
functions. Thus the Fourier coefficients of $G\left(\theta, \theta^{\prime}\right)\left|\frac{d \vec{r}}{d \theta}\right|$ can be computed according to (13) with spectral accuracy. For the term involving the logarithmic function (12), convolution sums will be used.

We now study the singularities in the normal derivative of the the Green's function $\frac{\partial G}{\partial n}$. The asymptotic expression of $H_{1}^{(1)}(\kappa R)$ for $\kappa R$ small has the following form[13]

$$
\begin{aligned}
H_{1}^{(1)}(\kappa R) & =J_{1}(\kappa R)+i Y_{1}(\kappa R) \\
& =J_{1}(\kappa R)+i\left(-\frac{2}{\pi \kappa R}+\frac{2}{\pi} \ln \left(\frac{\kappa R}{2}\right) J_{1}(\kappa R)+\text { odd powers of } \kappa R\right) \\
& =-\frac{2 i}{\pi \kappa R}+\frac{2 i}{\pi} \ln \left(\frac{\kappa R}{2}\right) J_{1}(\kappa R)+\text { odd powers of } \kappa R
\end{aligned}
$$

in which we have used the fact that the Bessel function of order unity, $J_{1}$, is a power series of odd power of $\kappa R$,

$$
J_{1}(\kappa R)=\frac{\kappa R}{2}-\frac{(\kappa R)^{3}}{16}+\cdots
$$

In addition, it can be shown that $\vec{R} \cdot \vec{n}=O\left(\left(\theta-\theta^{\prime}\right)^{2}\right)$. Thus it follows that

$$
\begin{aligned}
\frac{\partial G}{\partial n}\left(\theta, \theta^{\prime}\right) & =-\frac{i \kappa}{4}\left(-\frac{2 i}{\pi \kappa R}+\frac{2 i}{\pi} \ln \left(\frac{\kappa R}{2}\right) J_{1}(\kappa R)+\text { odd powers of } \kappa R\right) \frac{\vec{R} \cdot \vec{n}}{R} \\
& =\frac{\kappa}{2 \pi} \ln \left(\frac{\kappa R}{2}\right) J_{1}(\kappa R) \frac{\vec{R} \cdot \vec{n}}{R}+(\text { smooth terms })
\end{aligned}
$$

It is seen that, although $\frac{\partial G}{\partial n}$ is a finite function, it does not have smooth derivatives due to the logarithmic function appeared in the first term shown above. For this reason, its Fourier approximation will converge only at a finite rate of $1 / N^{3}$. To smooth out the function for computations by FFT, let

$$
\bar{H}\left(\theta, \theta^{\prime}\right) \equiv-\frac{i \kappa}{4} H_{1}^{(1)}(\kappa R) \frac{\vec{R} \cdot \vec{n}}{R}-\frac{\kappa}{2 \pi} \ln \left|2 \sin \left(\frac{\theta-\theta^{\prime}}{2}\right)\right| J_{1}(\kappa R) \frac{\vec{R} \cdot \vec{n}}{R}
$$

Then

$$
\frac{\partial G}{\partial n}\left(\theta, \theta^{\prime}\right)=\bar{H}\left(\theta, \theta^{\prime}\right)+\frac{\kappa}{2 \pi} \ln \left|2 \sin \left(\frac{\theta-\theta^{\prime}}{2}\right)\right| J_{1}(\kappa R) \frac{\vec{R} \cdot \vec{n}}{R}
$$


The functions $\bar{H}\left(\theta, \theta^{\prime}\right)$ and $J_{1}(\kappa R) \frac{\vec{R} \cdot \vec{n}}{R}$ in (14) are now periodic and infinitely differentiable. The Fourier coefficients of $\frac{\partial G}{\partial n}\left(\theta, \theta^{\prime}\right)\left|\frac{d \vec{r}}{d \theta}\right|$ will be computed according to (14).

Following the above analysis, we then express the boundary integral equation (10) as follows :

$$
\begin{gathered}
\frac{1}{2} \sum_{n=-N / 2}^{N / 2-1} \hat{\phi}_{n} e^{i n \theta^{\prime}}+\sum_{n=-N / 2}^{N / 2-1} \hat{\phi}_{n}\left(\int_{0}^{2 \pi} e^{i n \theta} \bar{H}\left(\theta, \theta^{\prime}\right)\left|\frac{d \vec{r}}{d \theta}\right| d \theta\right. \\
\left.+\frac{\kappa}{2 \pi} \int_{0}^{2 \pi} e^{i n \theta} \ln \left|2 \sin \left(\frac{\theta-\theta^{\prime}}{2}\right)\right| J_{1}(\kappa R) \frac{\vec{R} \cdot \vec{n}}{R}\left|\frac{d \vec{r}}{d \theta}\right| d \theta\right) \\
=\sum_{n=-N / 2}^{N / 2-1} \hat{\psi}_{n}\left(\int_{0}^{2 \pi} e^{i n \theta} \bar{G}\left(\theta, \theta^{\prime}\right)\left|\frac{d \vec{r}}{d \theta}\right| d \theta-\frac{1}{2 \pi} \int_{0}^{2 \pi} e^{i n \theta} \ln \left|2 \sin \left(\frac{\theta-\theta^{\prime}}{2}\right)\right| J_{0}(\kappa R)\left|\frac{d \vec{r}}{d \theta}\right| d \theta\right)
\end{gathered}
$$

Now all the integrals in (15) are in a form which can be evaluated numerically with spectral accuracy.

\subsection{Discretization}

In what follows, a spectral collocation approach will be taken in deriving the algebraic system for the boundary integral equation (15). Let us introduce two sets of discretization points (Figure 1)

$$
\begin{aligned}
\theta_{j} & =2 \pi j / N, \quad j=0,1, \ldots, N-1 \\
\theta_{j^{\prime}}^{\prime} & =2 \pi\left(j^{\prime}+\frac{1}{2}\right) / N, \quad j^{\prime}=0,1, \ldots, N-1
\end{aligned}
$$

The reason for using two sets of discretization points, as will be clear later, is that it avoids the direct evaluations of $\bar{G}\left(\theta, \theta^{\prime}\right)$ and $\bar{H}\left(\theta, \theta^{\prime}\right)$ at points where $\theta=\theta^{\prime}$. Although both functions are finite there, their limits are geometry dependent. The current discretization is robust. 
We then require that the boundary integral equation (15) be satisfied at collocation points $\theta^{\prime}=\theta_{j^{\prime}}^{\prime}, j^{\prime}=0,1, \ldots, N-1$.

For convenience of discussion, we denote the following Fourier series approximations at $\theta_{j^{\prime}}^{\prime}$ for $j^{\prime}=0,1, \ldots, N-1$,

$$
\begin{aligned}
\bar{G}\left(\theta, \theta_{j^{\prime}}^{\prime}\right)\left|\frac{d \vec{r}}{d \theta}\right| & =\sum_{n=-N / 2}^{N / 2-1} g_{j^{\prime} n} e^{-i n \theta} \\
\bar{H}\left(\theta, \theta_{j^{\prime}}^{\prime}\right)\left|\frac{d \vec{r}}{d \theta}\right| & =\sum_{n=-N / 2}^{N / 2-1} h_{j^{\prime} n} e^{-i n \theta} \\
{\left[J_{0}(\kappa R)\right]_{\theta_{j^{\prime}}}\left|\frac{d \vec{r}}{d \theta}\right| } & =\sum_{n=-N / 2}^{N / 2-1} p_{j^{\prime} n} e^{-i n \theta} \\
{\left[J_{1}(\kappa R) \frac{\vec{R} \cdot \vec{n}}{R}\right]_{\theta_{j^{\prime}}}\left|\frac{d \vec{r}}{d \theta}\right| } & =\sum_{n=-N / 2}^{N / 2-1} q_{j^{\prime} n} e^{-i n \theta}
\end{aligned}
$$

The coefficients of the expansions are calculated by FFT (backward in the usual sense) as follows:

$$
\begin{aligned}
& g_{j^{\prime} n}=\frac{1}{N} \sum_{j=0}^{N-1} e^{i n \theta_{j}} \bar{G}\left(\theta_{j}, \theta_{j^{\prime}}^{\prime}\right)\left|\frac{d \vec{r}}{d \theta}\left(\theta_{j}\right)\right| \\
& h_{j^{\prime} n}=\frac{1}{N} \sum_{j=0}^{N-1} e^{i n \theta_{j}} \bar{H}\left(\theta_{j}, \theta_{j^{\prime}}^{\prime}\right)\left|\frac{d \vec{r}}{d \theta}\left(\theta_{j}\right)\right| \\
& p_{j^{\prime} n}=\frac{1}{N} \sum_{j=0}^{N-1} e^{i n \theta_{j}}\left[J_{0}(\kappa R)\right]_{\theta_{j^{\prime}}}\left|\frac{d \vec{r}}{d \theta}\left(\theta_{j}\right)\right| \\
& q_{j^{\prime} n}=\frac{1}{N} \sum_{j=0}^{N-1} e^{i n \theta_{j}}\left[J_{1}(\kappa R) \frac{\vec{R} \cdot \vec{n}}{R}\right]_{\theta_{j^{\prime}}}\left|\frac{d \vec{r}}{d \theta}\left(\theta_{j}\right)\right|
\end{aligned}
$$

In addition, we denote (12) as

$$
\ln \left|2 \sin \left(\frac{\theta-\theta^{\prime}}{2}\right)\right|=\sum_{n=-\infty}^{\infty} a_{n} e^{-i n\left(\theta-\theta^{\prime}\right)}
$$

where $a_{0}=0$ and $a_{n}=-\frac{1}{2|n|}$ for $n \neq 0$. 
It is immediately seen that the two integrals involving $\bar{G}$ and $\bar{H}$ in (15) equal to $2 \pi g_{j^{\prime} n}$ and $2 \pi h_{j^{\prime} n}$ respectively. The other two integrals are obtainable by convolution sums. Specifically, using the definitions given in (16), we have

$$
\begin{aligned}
& u_{j^{\prime} n} \equiv \frac{1}{2 \pi} \int_{0}^{2 \pi} e^{i n \theta} \ln \left|2 \sin \left(\frac{\theta-\theta_{j^{\prime}}^{\prime}}{2}\right)\right| J_{0}(\kappa R)\left|\frac{d \vec{r}}{d \theta}\right| d \theta=\sum_{m=-N / 2}^{N / 2-1} p_{j^{\prime} m} a_{n-m} e^{i(n-m) \theta_{j^{\prime}}, \quad(17 a)} \\
& v_{j^{\prime} n} \equiv \frac{1}{2 \pi} \int_{0}^{2 \pi} e^{i n \theta} \ln \left|2 \sin \left(\frac{\theta-\theta_{j^{\prime}}^{\prime}}{2}\right)\right| J_{1}(\kappa R) \frac{\vec{R} \cdot \vec{n}}{R}\left|\frac{d \vec{r}}{d \theta}\right| d \theta=\sum_{m=-N / 2}^{N / 2-1} q_{j^{\prime} m} a_{n-m} e^{i(n-m)\left(\theta^{\prime} 77 b\right)}
\end{aligned}
$$

By substituting (16) and (17) into (15), the resulting algebraic system is :

$$
\frac{1}{2} \sum_{n=-N / 2}^{N / 2-1} \hat{\phi}_{n} e^{i n \theta_{j^{\prime}}^{\prime}}+\sum_{n=-N / 2}^{N / 2-1} \hat{\phi}_{n}\left(2 \pi h_{j^{\prime} n}+\kappa u_{j^{\prime} n}\right)=\sum_{n=-N / 2}^{N / 2-1} \hat{\psi}_{n}\left(2 \pi g_{j^{\prime} n}-v_{j^{\prime} n}\right)
$$

for $j^{\prime}=0,1,2, \ldots, N-1$.

Equation (18) is easily cast into a matrix form

$$
A \hat{\Phi}=B \hat{\Psi}
$$

where $\hat{\Phi}$ and $\hat{\Psi}$ are the vectors containing $\hat{\phi}_{n}$ and $\hat{\psi}_{n}$, respectively. For Dirichlet boundary condition, $\hat{\Psi}$ is solved from (19) with $\hat{\Phi}$ obtained from the boundary condition and vice versa for the Neumann boundary conditions. The Robin type impedance boundary condition can be treated similarly.

\subsection{Evaluation of convolution sums}

The convolution sums in (17) require $O(N)$ multiplications for each $u_{j^{\prime} n}$ and $v_{j^{\prime} n}$. Thus the total operations of obtaining the convolution sums are of order $O\left(N^{3}\right)$. This cost can be reduced considerably to $O\left(N^{2} \log _{2} N\right)$ by the use of a pseudospectral transformation method with de-aliasing techniques[6,7]. For completeness, the evaluation of (17) with a "padding" de-aliasing technique is given below.

Let $M \geq 3 N$ and

$$
\xi_{j}=2 \pi j / M, \quad j=0,1,2, \ldots M-1
$$


Compute the following using FFT for $j=0,1,2, \ldots M-1$ :

$$
\begin{aligned}
& A_{j^{\prime} j}=\sum_{m=-M / 2}^{M / 2-1} \tilde{a}_{m} e^{i m \theta_{j^{\prime}}^{\prime}} e^{-i m \xi_{j}} \\
& P_{j^{\prime} j}=\sum_{m=-M / 2}^{M / 2-1} \tilde{p}_{j^{\prime} m} e^{i m \theta_{j^{\prime}}^{\prime}} e^{-i m \xi_{j}}
\end{aligned}
$$

where

$$
\begin{gathered}
\tilde{a}_{m}=\left\{\begin{array}{cc}
a_{m} & -N \leq m \leq N-1 \\
0 & \text { other }
\end{array}\right. \\
\tilde{p}_{j^{\prime} m}=\left\{\begin{array}{cc}
p_{j^{\prime} m} & -N / 2 \leq m \leq N / 2-1 \\
0 & \text { other }
\end{array}\right.
\end{gathered}
$$

and form the product

$$
U_{j^{\prime} j}=A_{j^{\prime} j} P_{j^{\prime} j}
$$

Then the convolution sum $u_{j^{\prime} n}$ is the (backward) FFT of $U_{j^{\prime} j}$ :

$$
u_{j^{\prime} n}=\frac{1}{M} \sum_{j=0}^{M-1} U_{j^{\prime} j} e^{i n \xi_{j}}
$$

for $-N / 2 \leq n \leq N / 2-1$. The convolution sum $v_{j^{\prime} n}$ can be obtained identically.

\section{NUMERICAL EXAMPLE : SCATTERING OF A PLANE WAVE BY AN ELLIPTIC CYLINDER}

The numerical method described in the previous section will now be applied to the problem of a plane wave scattering by an elliptic cylinder. Exact solutions of wave scattering by an elliptic cylinder can be easily obtained in infinite series[12]. However, numerical evaluation of the exact infinite series is not easily obtainable due to the complexity of the Mathieu functions involved. Our purpose here is to demonstrate the exponential rate of convergence of the spectral method formulated in the previous 
section. The numerical results will also be compared with some asymptotic values available in the literature[14].

\subsection{Spectral rate of convergence}

Let the plane incident wave having an incident angle $\alpha$ about the $x$ axis be

$$
\phi_{i}=e^{i \kappa(x \cos \alpha+y \sin \alpha)}
$$

The scattered wave, $\phi$, satisfies the Helmholtz equation (1). The boundary conditions considered here are the Dirichlet (soft) type $\phi=-\phi_{i}$ or the Neumann (hard) type $\frac{\partial \phi}{\partial n}=-\frac{\partial \phi_{i}}{\partial n}$.

A simple parameterization of the elliptic cylinder is given by

$$
\Gamma: \quad(x, y)=(a \cos \theta, b \sin \theta), \quad 0 \leq \theta \leq 2 \pi
$$

where $a$ and $b$ denote the major and minor axis of the ellipse. The normal vector on $\Gamma$ is $\vec{n}=-(b \cos \theta, a \sin \theta) / \sqrt{b^{2} \cos ^{2} \theta+a^{2} \sin ^{2} \theta}$.

Numerical results for the Dirichlet boundary condition will be presented first. For this calculation the parameters have been taken to be $a=2, b=1$ and $\kappa=2 \pi$. In table I, the values of the solution at three selected points on the boundary are given as the number of collocation points increases. Listed are the values of $\left|\frac{\partial \phi}{\partial n}\right|$ at $\theta=0^{\circ}$, $90^{\circ}$ and $180^{\circ}$ on the boundary for the incident angle $\alpha=0$. Since no exact value is available, the numerical solutions are compared with the results obtained for $N=256$. Clearly exponential rate of convergence is observed.

We point out that although the parameterization of the ellipse given in (20) is a convenient one, it may not the be the best. For example, the following form of parameterization, which has more uniformly distributed collocation points than (20),

$$
\Gamma: \quad(x, y)=(a \cos (\theta+0.05 \sin (2 \theta)), b \sin (\theta+0.05 \sin (2 \theta))), \quad 0 \leq \theta \leq 2 \pi
$$

yields the values shown in Table II. A somewhat better accuracy for small $N$ is observed. 
In Figure 2 the solution, $\frac{\partial \phi}{\partial n}$, as a function of $\theta$ on the surface of the elliptic cylinder has been plotted. Shown are the results for the number of collocation points being 32 and 256.

\subsection{Far field directivity}

Far field scattered intensities, computed as $r \phi^{2}$, have also been calculated. The values on an exterior point are obtained through the boundary integral as

$$
\begin{aligned}
\phi(\vec{r}) & =\int_{\Gamma}\left(G \frac{\partial \phi}{\partial n}-\phi \frac{\partial G}{\partial n}\right) d \Gamma \\
& =\sum_{n=-N / 2}^{N / 2-1} \hat{\psi}_{n} \int_{0}^{2 \pi} e^{i n \theta} G\left|\frac{d \vec{r}}{d \theta}\right| d \theta-\sum_{n=-N / 2}^{N / 2-1} \hat{\phi}_{n} \int_{0}^{2 \pi} e^{i n \theta} \frac{\partial G}{\partial n}\left|\frac{d \vec{r}}{d \theta}\right| d \theta
\end{aligned}
$$

The Green's function for points lying outside of the boundary does not have any singularity. Thus (22) is evaluated using FFT directly. The directivities of the scattered intensity for wave incident angle $\alpha=0^{\circ}, 30^{\circ}, 60^{\circ}$ and $90^{\circ}$ are shown in Figure 3. For this calculation, $a=2, b=1$ and $\kappa=2 \pi$.

Barakat[14] gives some asymptotic values of long wave scattering of an elliptic cylinder at far field for normal incidence. A comparison of the current numerical results with the asymptotic values is presented in Tables III and IV. For these cases, $a=\cosh \mu_{0}, b=\sinh \mu_{0}$ and $\kappa=0.2$. Here $\mu_{0}$ corresponds to a value in the elliptic coordinates. The results of the far field scattered intensity for $\mu_{0}=1.0,0.6$ and 0.2 are presented in Table III and IV for the Dirichlet and Neumann boundary conditions, respectively. For the present long wave scattering, $N=64$ has been used for all the calculations. The numerical computation agrees with the asymptotic estimation.

Finally we note that although the exterior scattering problem is uniquely determined, the direct formulation of the Boundary Integral Equation used here would not yield a unique solution when the wave number $\kappa$ coincides with the eigenvalues of a correspondent interior homogeneous problem. On the other hand, this numerical difficulty is well understood and remedies are readily available[10,11]. For example, unique 
solution can be obtained in a combination of single- and double-layer formulation of the Boundary Integral Equation[15]. The modifications of the present spectral implementation to other formulations of the Boundary Integral Equation are straightforward.

\section{CONCLUSIONS}

In this paper, a spectral method of solving the Boundary Integral Equation is presented. It is shown that the integral kernels for the Helmholtz equation contain singular terms that have to be removed to achieve the spectral accuracy. Detailed numerical implementation of a Fourier collocation formulation has been given. The non-smoothness of the integration kernels is subtracted out by using a logarithmic function whose Fourier expansion is known. The numerical formulation presented here preserves the spectral accuracy and yields an exponential rate of convergence.

Compared to the Boundary Element approaches, the Spectral Boundary Integral Equation Method presented in this paper would yield matrices of much smaller size since the latter requires far fewer points to achieve the same accuracy. This, of course, reduces the complexity and the cost of solving the resultant algebraic system. Since both methods will result in dense matrices, it appears that the spectral formulation is more advantageous for problems with smooth geometries.

\section{ACKNOWLEDGMENTS}

The author wishes to thank Drs. J. Webb and D.G. Lasseigne for helpful comments.

\section{REFERENCES}

1. C. A. Brebbia and M. S. Ingber, Boundary Element Technology VII, Elsevier, 1992.

2. R. D. Ciskowski and C. A. Brebbia, Boundary Element Methods in Acoustics, Computational Mechanics Publications, 1991. 
3. P. C. Waterman, "New formulation of Acoustic Scattering," J. Acoust. Soc. Amer. 45(6), 1417-1429, 1969.

4. V. K. Varadan and V. V. Varadan, Acoustic, Electromagnetic and Elastic Wave Scattering - Focus on the T-Matrix Approach, Pergamon Press, 1980.

5. W. Tobocman, "Comparison of the T-matrix and Helmholtz integral equation methods for wave scattering calculations", J. Acoust. Soc. Amer. 77(2), 369-374, 1985.

6. D. Gottlieb and S. Orszag, Numerical Analysis of Spectral Methods : Theory and Applications, SIAM, 1977.

7. C. Canuto, M. Y. Hussaini, A. Quarteroni and T. A. Zang, Spectral Methods in Fluid Dynamics, Springer-Verlag, 1988.

8. N. N. Bojarski, "Scattering by a cylinder: A fast exact numerical solution," J. Acoust. Soc. Amer. 75(2), 320-323, 1984.

9. G. T. Schuster, "A fast exact numerical solution for the acoustic response of concentric cylinders with penetrable interfaces." J. Acoust. Soc. Amer. 87(2), 495-502, 1990.

10. D. Colton and R. Kress, Integral Equation Methods In Scattering Theory, Wiley Interscience, 1983.

11. G. Chen and J. Zhou, Boundary Element Methods, Academic Press, 1992.

12. P. Morse and H. Feshbach, Methods of Theoretical Physics, McGraw-Hill, 1953.

13. M. Abramowitz and I. A. Stegun, Handbook of Mathematical Functions, Dover, 1965.

14. R. Barakat, "Diffraction of plane waves by an elliptic cylinder," J. Acoust. Soc. Amer. 35(12), 1990-1996, 1963.

15. A. J. Burton and G. F. Miller, "The application of integral equation methods to the numerical solution of some exterior boundary-value problems", Proc. Roy. Soc. Lond. A, 323, 201-210, 1971. 


\section{TABLE I}

Values of $\left|\frac{\partial \phi}{\partial n}\right|$ for the Dirichlet boundary condition at selected points on the boundary.

The boundary is given by (20).

$a=2, b=1, \kappa=2 \pi$, incident angle $\alpha=0^{\circ}$.

\begin{tabular}{|c|c|c|c|c|}
\hline $\mathrm{N}$ & $\theta=0^{\circ}$ & $\theta=90^{\circ}$ & $\theta=180^{\circ}$ & Relative Error \\
\hline 32 & 6.178009567 & 2.376671291 & 7.134225119 & 0.04 \\
\hline 48 & 6.379285016 & 2.175324633 & 6.914464114 & $0.2 \times 10^{-4}$ \\
\hline 64 & 6.379334010 & 2.175259123 & 6.914387795 & $0.1 \times 10^{-7}$ \\
\hline 80 & 6.379333962 & 2.175259114 & 6.914387773 & $10^{-9}$ \\
\hline 96 & 6.379333961 & 2.175259114 & 6.914387772 & $10^{-11}$ \\
\hline 256 & 6.379333961 & 2.175259114 & 6.914387772 & \\
\hline
\end{tabular}

\section{TABLE II}

Values of $\left|\frac{\partial \phi}{\partial n}\right|$ for the Dirichlet boundary condition at selected points on the boundary.

The boundary is given by (21).

$$
a=2, b=1, \kappa=2 \pi \text {, incident angle } \alpha=0^{\circ} \text {. }
$$

\begin{tabular}{|c|c|c|c|c|}
\hline $\mathrm{N}$ & $\theta=0^{\circ}$ & $\theta=90^{\circ}$ & $\theta=180^{\circ}$ & Relative Error \\
\hline 32 & 6.402635098 & 2.165098187 & 6.899520927 & 0.005 \\
\hline 48 & 6.379355527 & 2.175263759 & 6.914393819 & $0.3 \times 10^{-5}$ \\
\hline 64 & 6.379334276 & 2.175259159 & 6.914387893 & $0.5 \times 10^{-7}$ \\
\hline 80 & 6.379333966 & 2.175259114 & 6.914387774 & $0.5 \times 10^{-9}$ \\
\hline 96 & 6.379333961 & 2.175259114 & 6.914387772 & $10^{-11}$ \\
\hline 256 & 6.379333961 & 2.175259114 & 6.914387772 & - \\
\hline
\end{tabular}


TABLE III

Values of $r \phi^{2}$ at far field for the selected angles. Dirichlet type soft wall boundary conditions are applied.

$a=\cosh \mu_{0}, b=\sinh \mu_{0}, \kappa=0.2$ and incident angle $\alpha=90^{\circ}$.

\begin{tabular}{|c|c|c|}
\hline Far Field Angle & Numerical & Asymptotic[14] \\
\hline$\mu_{0}=1.0, \theta=0^{\circ}, 180^{\circ}$ & 1.769057417 & 1.774 \\
\hline$\mu_{0}=1.0, \theta=90^{\circ}$ & 2.137413140 & 2.113 \\
\hline$\mu_{0}=1.0, \theta=270^{\circ}$ & 1.538098511 & 1.491 \\
\hline$\mu_{0}=0.6, \theta=0^{\circ}, 180^{\circ}$ & 1.364415306 & 1.367 \\
\hline$\mu_{0}=0.6, \theta=90^{\circ}$ & 1.506576730 & 1.484 \\
\hline$\mu_{0}=0.6, \theta=270^{\circ}$ & 1.287260683 & 1.258 \\
\hline$\mu_{0}=0.2, \theta=0^{\circ}, 180^{\circ}$ & 1.062775602 & 1.064 \\
\hline$\mu_{0}=0.2, \theta=90^{\circ}$ & 1.107062467 & 1.087 \\
\hline$\mu_{0}=0.2, \theta=270^{\circ}$ & 1.062022484 & 1.041 \\
\hline
\end{tabular}


TABLE IV

Values of $r \phi^{2}$ at far field for the selected angles. Neumann type hard wall boundary conditions are applied.

$a=\cosh \mu_{0}, b=\sinh \mu_{0}, \kappa=0.2$ and incident angle $\alpha=90^{\circ}$.

\begin{tabular}{|c|c|c|}
\hline Far Field Angle & Numerical & Asymptotic[14] \\
\hline$\mu_{0}=1.0, \theta=0^{\circ}, 180^{\circ}$ & 0.009175011010 & 0.00884 \\
\hline$\mu_{0}=1.0, \theta=90^{\circ}$ & 0.02258893859 & 0.0216 \\
\hline$\mu_{0}=1.0, \theta=270^{\circ}$ & 0.1110188572 & 0.1113 \\
\hline$\mu_{0}=0.6, \theta=0^{\circ}, 180^{\circ}$ & 0.001676516587 & 0.00165 \\
\hline$\mu_{0}=0.6, \theta=90^{\circ}$ & 0.007209616977 & 0.0071 \\
\hline$\mu_{0}=0.6, \theta=270^{\circ}$ & 0.02745548914 & 0.0273 \\
\hline$\mu_{0}=0.2, \theta=0^{\circ}, 180^{\circ}$ & 0.0001288604229 & 0.00013 \\
\hline$\mu_{0}=0.2, \theta=90^{\circ}$ & 0.003710427872 & 0.0037 \\
\hline$\mu_{0}=0.2, \theta=270^{\circ}$ & 0.006989684179 & 0.0069 \\
\hline
\end{tabular}



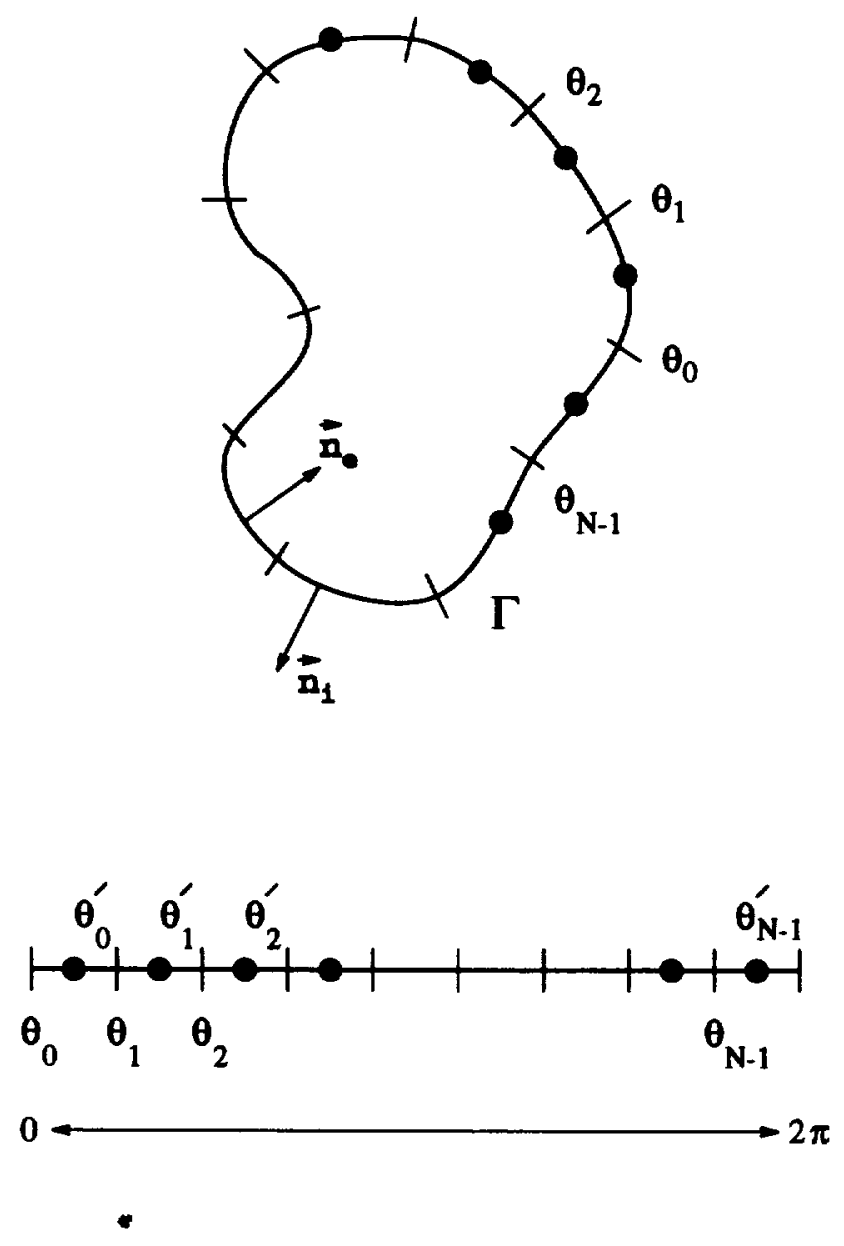

Figure 1. Schematic of discretization of the boundary. $\theta$ is the parameterizing variable for the boundary contour. $\vec{n}_{e}$ and $\vec{n}_{i}$ indicate the direction of the normal vector for an exterior and interior problem respectively. 

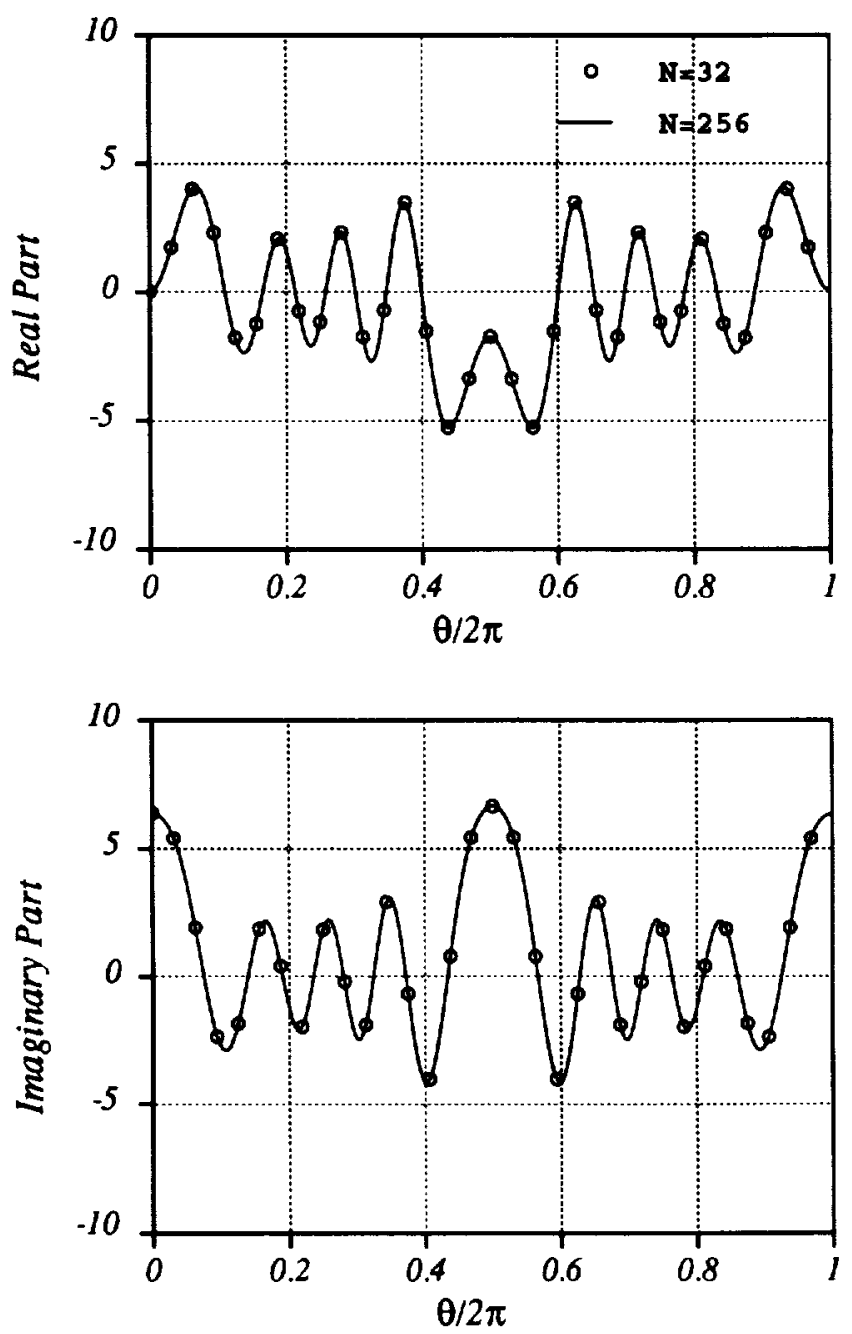

Figure 2. Solution $\frac{\partial \phi}{\partial n}$ on the surface of the ellipse for the Dirichlet boundary condition. $a=2, b=1$ and $\kappa=2 \pi$. Plane wave incident angle $\alpha=0^{\circ}$. The parameterization of the ellipse is that of (21). 

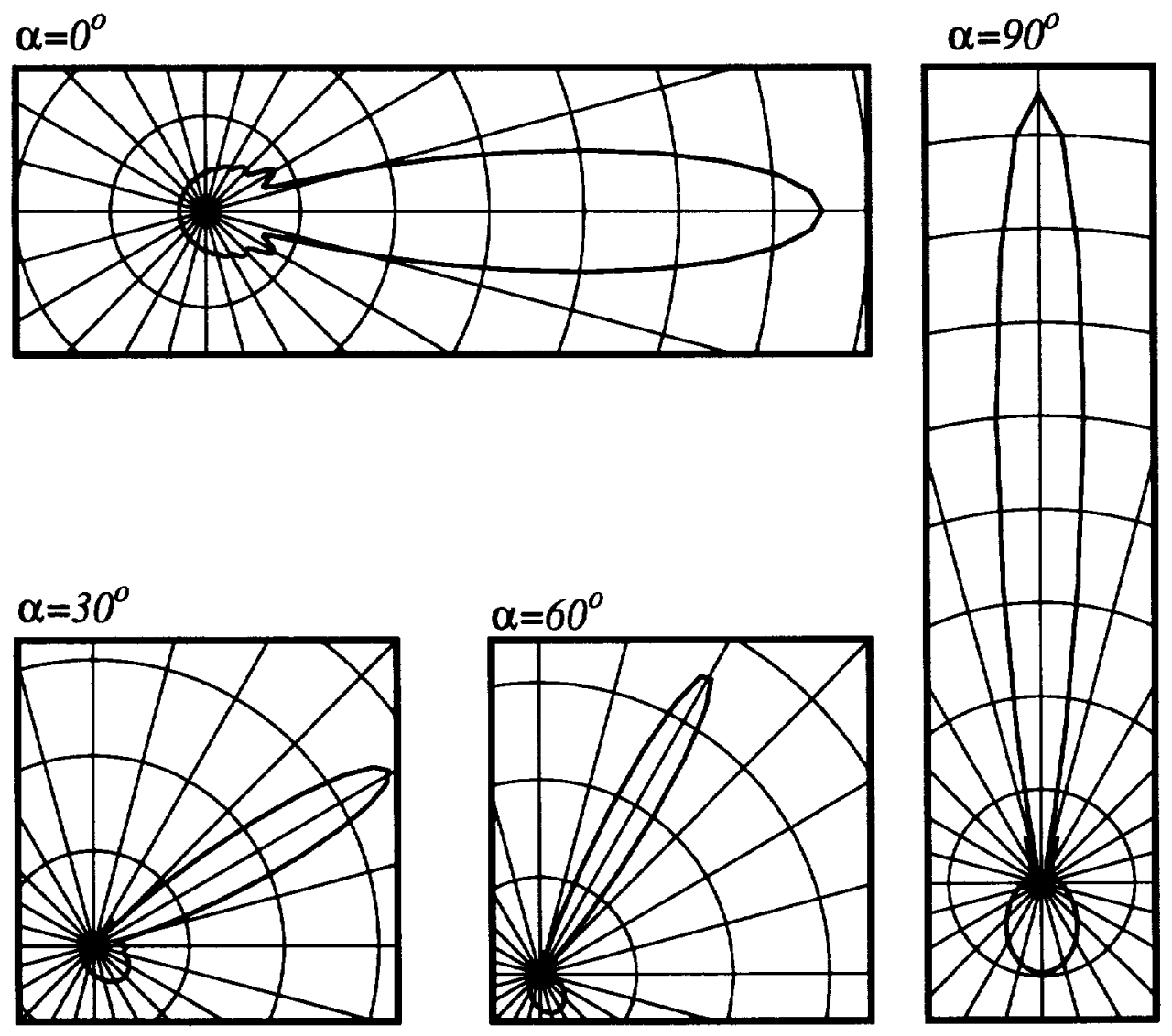

Figure 3. Far field directivities of the scattered wave for indicated incident angles. $a=2, b=1$ and $\kappa=2 \pi$, with Dirichlet boundary condition. 


\begin{tabular}{|c|c|c|}
\hline \multicolumn{2}{|c|}{ REPORT DOCUMENTATION PAGE } & $\begin{array}{l}\text { Form Approved } \\
\text { OMB No. 0704-0188 }\end{array}$ \\
\hline \multicolumn{3}{|c|}{ 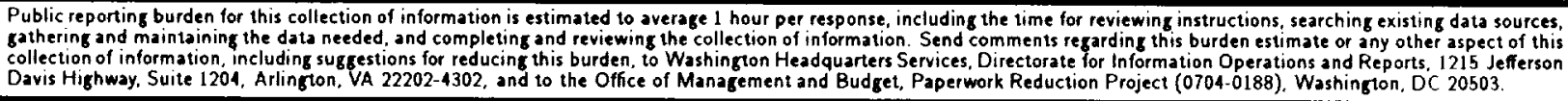 } \\
\hline \multicolumn{2}{|c|}{\begin{tabular}{|l|l|} 
1. AGENCY USE ONLY(Leave blank) & $\begin{array}{l}\text { 2. REPORT DATE } \\
\text { March } 1994\end{array}$ \\
\end{tabular}} & $\begin{array}{l}\text { 3. REPORT TYPE AND DATES COVERED } \\
\text { Contractor Report }\end{array}$ \\
\hline \multicolumn{2}{|c|}{$\begin{array}{l}\text { 4. TITLE AND SUBTITLE } \\
\text { A SPECTRAL BOUNDARY INTEGRAL EQUATION METHOD } \\
\text { FOR THE 2-D HELMHOLTZ EQUATION }\end{array}$} & $\begin{array}{l}\text { C NAS1-19480 } \\
\text { WU 505-90-52-01 }\end{array}$ \\
\hline \multicolumn{2}{|l|}{$\begin{array}{l}\text { 6. AUTHOR(S) } \\
\text { Fang Q. Hu }\end{array}$} & \\
\hline \multicolumn{2}{|c|}{$\begin{array}{l}\text { 7. PERFORMING ORGANIZATION NAME(S) AND ADDRESS(ES) } \\
\text { Institute for Computer Applications in Science } \\
\text { and Engineering } \\
\text { Mail Stop 132C, NASA Langley Research Center } \\
\text { Hampton, VA 23681-0001 }\end{array}$} & $\begin{array}{l}\text { 8. PERFORMING ORGANIZATION } \\
\text { REPORT NUMBER } \\
\text { ICASE Report No. } 94-15\end{array}$ \\
\hline \multicolumn{2}{|c|}{$\begin{array}{l}\text { 9. SPONSORING/MONITORING AGENCY NAME(S) AND ADDRESS(ES) } \\
\text { National Aeronautics and Space Administration } \\
\text { Langley Research Center } \\
\text { Hampton, VA 23681-0001 }\end{array}$} & $\begin{array}{l}\text { 10. SPONSORING/MONITORING } \\
\text { AGENCY REPORT NUMBER } \\
\text { NASA CR-194890 } \\
\text { ICASE Report No. } 94-15\end{array}$ \\
\hline \multicolumn{3}{|c|}{$\begin{array}{l}\text { 11. SUPPLEMENTARY NOTES } \\
\text { Langley Technical Monitor: Michael F. Card } \\
\text { Final Report } \\
\text { To be submitted to Journal of Computational Physics }\end{array}$} \\
\hline \multicolumn{2}{|c|}{$\begin{array}{l}\text { 12a. DISTRIBUTION/AVAILABILITY STATEMENT } \\
\text { Unclassified-Unlimited } \\
\text { Subject Category } 64,34\end{array}$} & 12b. DISTRIBUTION CODE \\
\hline \multicolumn{3}{|c|}{$\begin{array}{l}\text { 13. ABSTRACT (Maximum } 200 \text { words) } \\
\text { In this paper,we present a new numerical formulation of solving the Boundary Integral Equations reformulated from } \\
\text { the Helmholtz equation. The boundaries of the problems are assumed to be smooth closed contours. The solution } \\
\text { on the boundary is treated as a periodic function, which is in turn approximated by a truncated Fourier series. } \\
\text { A Fourier collocation method is followed in which the boundary integral equation is transformed into a system } \\
\text { of algebraic equations. It is shown that in order to achieve spectral accuracy for the numerical formulation, the } \\
\text { non-smoothness of the integral kernels, associated with the Helmholtz equation, must be carefully removed. The } \\
\text { emphasis of the paper is on investigating the essential elements of removing the nonsmoothness of the integral kernels } \\
\text { in the spectral implementation. The present method is robust for a general boundary contour. Aspects of efficient } \\
\text { implementation of the method using FFT are also discussed. A numerical example of wave scattering is given in } \\
\text { which the exponential accuracy of the present numerical method is demonstrated. }\end{array}$} \\
\hline
\end{tabular}

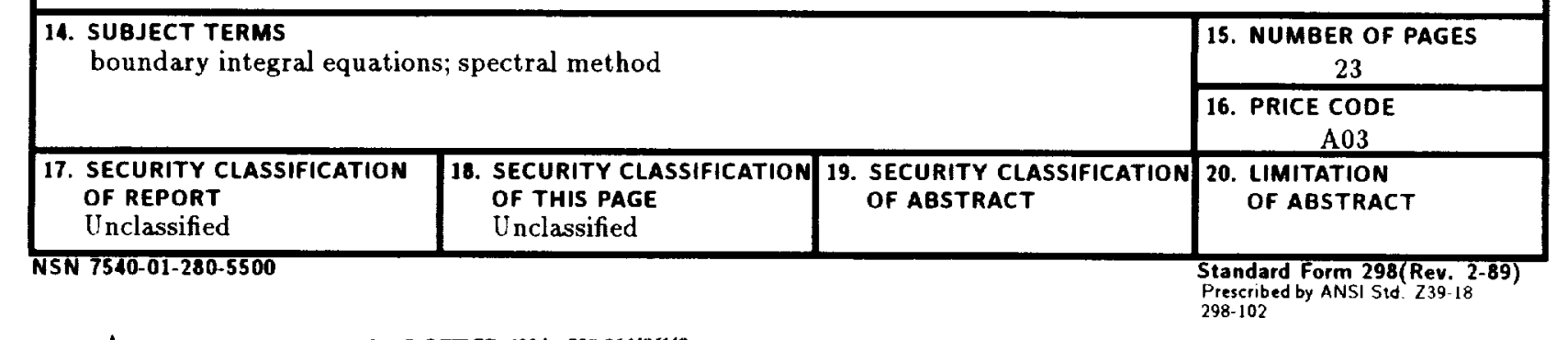

A U.S. GOVERNMENT PRINTING OFFICE: 1994 - 522-06//664 